\title{
Multilocus sequence analysis of Borrelia burgdorferi s.l. in Russia
}

\author{
Tatyana A. Mukhacheva, Sergey Y. Kovalev* \\ Laboratory of Molecular Genetics, Department of Biology, Ural Federal University, Lenin Avenue 51, Yekaterinburg 620000, Russia
}

\section{A R T I C L E I N F O}

\section{Article history:}

Received 27 December 2012

Received in revised form 15 February 2013

Accepted 19 February 2013

Available online 18 April 2013

\section{Keywords:}

Borrelia

MLST

rrf-rrl intergenic spacer

NT29 group

\begin{abstract}
A B S T R A C T
Borrelia burgdorferi sensu lato is a species complex that includes the causative agents of Lyme borreliosis (LB). Classification of the complex was greatly influenced by the method of multilocus sequence typing (MLST), proposed in 2008 and analyzing the spatial distribution of sequence types (STs). Despite the fact that Russia is the largest natural focus of LB to date, it is represented by only 3 strains of the 1323 strains deposited in the MLST database. In this paper, we identified STs for 24 B. burgdorferi s.l. strains isolated from ticks from almost all regions of Russia, 16 of which have not been described so far. It has been shown that the Russian isolates of $B$. afzelii are of Asian origin and are characterized by a lack of territorial mixing of STs. In contrast, $B$. garinii and B. bavariensis showed ST mixing between different localities. Comparison of MLST data with the results of sequence analysis of rrf-rrl intergenic spacer led to the conclusion that the previously described genomic groups of $B$. garinii correspond to the genospecies according to the new classification: 20047 corresponds to B. garinii, and NT29 corresponds to B. bavariensis. The genomic group, ChY13p, characterized by an unusual PCR-RFLP profile, belongs to the species $B$. garinii (prototype strain 20047). Thus, the use of a reliable method to study the phylogeny and evolution of Borrelia based on MLST, helps to clarify the existing classification. The standardized research procedure and database created could become the basis for a global scientific cooperation in LB research.
\end{abstract}

(c) 2013 Elsevier GmbH. All rights reserved.

\section{Introduction}

Borrelia burgdorferi sensu lato is the causative agent of Lyme borreliosis (LB), a multisystem zoonotic disease. LB is registered in 72 regions of Russia with an annual incidence of 6400-9900 cases (Yastrebov et al., 2012). To date, at least 18 species (15 confirmed and 3 proposed) of $B$. burgdorferi sensu lato are known (Margos et al., 2011). They are differentiated by molecular genetic techniques. In particular, 2 genospecies (B. bavariensis, B. kurtenbachii) were proposed on the basis of multilocus sequence typing (MLST) and ecological traits (Margos et al., 2009, 2010). This method comprises the analysis of the nucleotide sequences of the fragments of conserved housekeeping genes and is widely used in the study of the genetic structure of populations and evolutionary processes within many bacterial pathogens (Enright and Spratt, 1999; Sullivan et al., 2005; Margos et al., 2008). The basic unit of analysis is a sequence type (ST), a sequence of 8 gene fragments that differ from each other by at least one nucleotide. In addition, there is an international database (http://borrelia.mlst.net/) containing the sequences of STs as well as epidemiological data and software for analysis. This makes MLST the most promising and objective method for studying Borrelia populations. However, among 1323 $B$. burgdorferi s.l. strains, whose sequences are deposited in the

\footnotetext{
* Corresponding author. Tel.: +7 343 2616826; fax: +7 3433507401.

E-mail address: Sergey.Kovalev@usu.ru (S.Y. Kovalev).
}

database, only 3 originated from Russia, but were deposited by Japanese researchers (Takano et al., 2011). Thus, despite the fact that the taiga zone of Russia, where Europe meets Asia, is the biggest natural focus of LB, this approach in molecular epidemiology of LB is still not used. However, the researchers emphasized the need to study Russian strains of Borrelia which is a key point in understanding the evolution and spread of Borrelia through Eurasia (Margos et al., 2011).

Historically, PCR-RFLP of the $\operatorname{rrf}(5 S)-\mathrm{rrl}$ (23S) intergenic spacer (Postic et al., 1994) was the first technique extensively used for the differential diagnosis of Borrelia genospecies circulating in Russia (B. burgdorferi s.s., B. afzelii, and B. garinii). This approach allowed the differentiation of the above genospecies as well as 3 genomic groups within B. garinii, differing by the profiles of restriction fragments (names are given according to the prototype strain): 20047 , NT29, and ChY13p (Postic et al., 1994; Li et al., 1998) and 2 groups within B. afzelii: VS461 and NT28 (Masuzawa et al., 1996). With the development of the MLST scheme, a long-needed clarification of the taxonomic status of these groups can be accomplished and may help unifying the scientific research carried out in different countries.

\section{Materials and methods}

Both Borrelia strain cultures and uncultured isolates from Ixodes persulcatus (Schulze, 1930) ticks were used in the study (Table 1). Eleven strains were obtained from ticks collected in the 
Table 1

Information about the strains used for the analysis.

\begin{tabular}{|c|c|c|c|c|c|c|}
\hline No. & Name & ST & Species & Origin $^{\mathrm{a}}$ & Strain/isolate & Year of isolation \\
\hline 1 & SPb8-08 & 438 & B. afzelii & Saint-Petersburg, north-western Russia (1) & Isolate & 2008 \\
\hline 2 & Krg1090-09 & 128 & B. bavariensis & Kurgan, West Siberia (9) & Isolate & 2009 \\
\hline 3 & Ekb166-10 & 441 & B. bavariensis & Sysert', Yekaterinburg region, Middle Urals (8) & Isolate & 2010 \\
\hline 4 & Ekb701-11G & 431 & B. garinii & Yekaterinburg, Middle Urals (7) & Strain & 2011 \\
\hline 5 & Ekb704-11G & 364 & B. garinii & Yekaterinburg, Middle Urals (7) & Strain & 2011 \\
\hline 6 & Ekb706-11G & 431 & B. garinii & Yekaterinburg, Middle Urals (7) & Strain & 2011 \\
\hline 7 & Ekb709-11 & 431 & B. garinii & Yekaterinburg, Middle Urals (7) & Isolate & 2011 \\
\hline 8 & Ekb712-11G & 154 & B. garinii & Yekaterinburg, Middle Urals (7) & Strain & 2011 \\
\hline 9 & Ekb767-11A & 432 & B. afzelii & Severouralsk, Yekaterinburg region, Middle Urals (6) & Strain & 2011 \\
\hline 10 & Prm7564-11G & 433 & B. bavariensis & Cherdyn', Perm region, Middle Urals (4) & Strain & 2011 \\
\hline 11 & Prm7565-11A & 434 & B. afzelii & Cherdyn', Perm region, Middle Urals (4) & Strain & 2011 \\
\hline 12 & Prm7567-11A & 434 & B. afzelii & Cherdyn', Perm region, Middle Urals (4) & Strain & 2011 \\
\hline 13 & Prm7569-11G & 374 & B. bavariensis & Cherdyn', Perm region, Middle Urals (4) & Strain & 2011 \\
\hline 14 & Prm7570-11A & 435 & B. afzelii & Cherdyn', Perm region, Middle Urals (4) & Strain & 2011 \\
\hline 15 & Alt730-11 & 439 & B. afzelii & Altai region, South Siberia (10) & Isolate & 2011 \\
\hline 16 & Krv750-11 & 436 & B. bavariensis & Omutninsk, Kirov region, European part of Russia (3) & Isolate & 2011 \\
\hline 17 & Alt756-11 & 436 & B. bavariensis & Altai region, South Siberia (10) & Isolate & 2011 \\
\hline 18 & Alt763-11 & 440 & B. bavariensis & Altai region, South Siberia (10) & Isolate & 2011 \\
\hline 19 & Vld836-11 & 437 & B. afzelii & Vladivostok, Far East (11) & Isolate & 2011 \\
\hline 20 & Arh948-12 & 443 & B. afzelii & Kotlas, Arkhangelsk region, north-western Russia (2) & Strain & 2012 \\
\hline 21 & Arh974-12 & 444 & B. afzelii & Kotlas, Arkhangelsk region, north-western Russia (2) & Isolate & 2012 \\
\hline 22 & Arh976-12 & 445 & B. bavariensis & Kotlas, Arkhangelsk region, north-western Russia (2) & Isolate & 2012 \\
\hline 23 & Arh997-12 & 446 & B. afzelii & Kotlas, Arkhangelsk region, north-western Russia (2) & Isolate & 2012 \\
\hline 24 & Prm7050-12 & 442 & B. afzelii & Kungur, Perm region, Middle Urals (5) & Isolate & 2012 \\
\hline
\end{tabular}

ST, sequence types.

a The numbers in parenthesis correspond to numbers of collection sites shown in Fig. 1.

surroundings of Yekaterinburg (Sverdlovsk region) and Cherdyn (Perm region), by culturing them in BSK-H medium. Additionally, sequences of 16 Borrelia isolates were obtained directly from ticks collected in St. Petersburg, Arkhangelsk, Kirov, Sverdlovsk, Altai, and Primorye regions (Fig. 1). Multilocus typing was performed according to the common MLST scheme (Margos et al., 2008) with minor modifications. Nucleotide sequences of 8 housekeeping gene fragments, total length $4785 \mathrm{bp}$, were identified. Sequences of rrf-rrl intergenic spacer were obtained for the same strains and isolates according to the standard procedure (Postic et al., 1994).

Nucleic acid was extracted using a Ribo-Sorb kit for RNA/DNA extraction (Interlabservis, Russia), and reverse transcription was performed using the Reverta-L kit (Interlabservis), both according to the manufacturer's instructions. Detection of Borrelia in ticks was performed by real-time PCR on an ABI PRIZM 7500 (Applied Biosystems, USA) according to the method by Ornstein and Barbour (2006). The amplification reaction for subsequent sequencing was performed in a Veriti ${ }^{\circledR}$ 60-Well Thermal Cycler (Applied Biosystems). PCR products were separated by electrophoresis on $2 \%$ agarose gel, and determination of the sequences of fragments was performed on the ABI PRIZM 310 Genetic Analyzer (Applied Biosystems). Sequences obtained for 10 strains and 14 isolates were deposited in GenBank under the numbers JX971230-JX971421

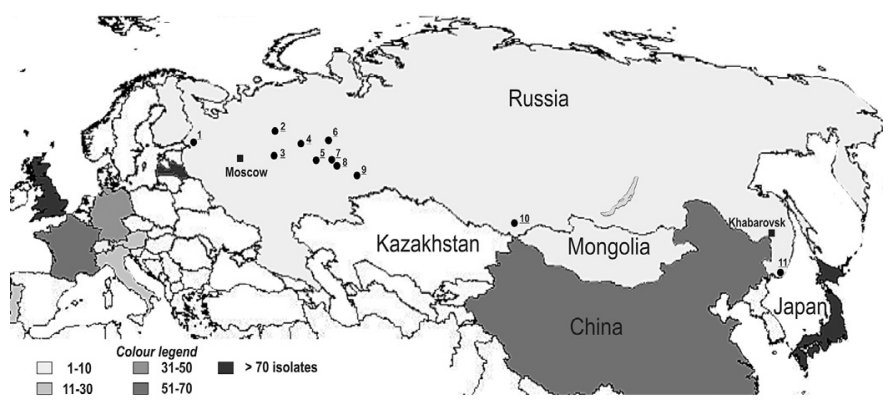

Fig. 1. Number of B. burgdorferi s.l. strains from different countries (shown by color, data are obtained from the MLST Database). Collection sites of samples obtained in the present study are indicated with circles and those of samples deposited in the database Borrelia MLST, with squares. Locations are numbered according to Table 1.
(MLST data) and KC261424-KC261447 (rrf-rrl intergenic spacer). Sequences from the international databases GenBank and Borrelia MLST (borrelia.mlst.net/) were included in the analysis. Phylogenetic analysis was performed using the software, MEGA v.5.5 (Tamura et al., 2011).

\section{Results}

Of 27 Borrelia-positive samples selected for MLST, 3 samples showed the presence of mixed-base peaks [intra- $(n=2)$ or interspecific $(n=1)$ mixed infections] and were excluded from the further analysis. Thus, we compared phylogenetic information present in genome fragments of 8 housekeeping genes (MLST) and the rrf-rrl intergenic spacer for 24 Borrelia strains and isolates from different regions of Russia, from St. Petersburg to Vladivostok (Fig. 1, Table 1, detailed information in Supplemental Table 1 ). Each of the obtained sequences belonged to one of 3 Borrelia genospecies, B. afzelii (11 strains), B. garinii (5), and $B$. bavariensis sp. nov. (8). Sixteen MLST STs obtained had not been present in the database Borrelia MLST and were newly described (STs 431-446). The sequences of 4 strains belonged to existing STs - ST128 (China, Japan, Mongolia), ST154 (China), ST364 (Japan), ST374 (Russia, Moscow region) (Fig. 2, Table 1). Genetic distances between the sequences obtained and those of type strains are given in Supplemental Table 2 .

Supplementary material related to this article found, in the online version, at doi:10.1016/j.ttbdis.2013.02.004.

Phylogenetic trees were constructed using rrf-rrl intergenic spacer sequences and concatenated sequences of housekeeping genes (Fig. 3). Comparative analysis indicates that the 2 different approaches result in similar topologies, but bootstrap values for the tree obtained with MLST data are greater, proving it to be more reliable (Fig. 3). Generally, the previously described genomic groups of $B$. garinii correspond to genospecies according to the new classification: 20047 indicates B. garinii, and NT29 clusters with B. bavariensis sp. nov. The most significant difference between the phylogenies was the position of the genomic group ChY13p, whose specific PCRRFLP sequence was more similar to those of $B$. afzelii (Livanova et al., 2003). However, analysis of the housekeeping genes (MLST) proved 


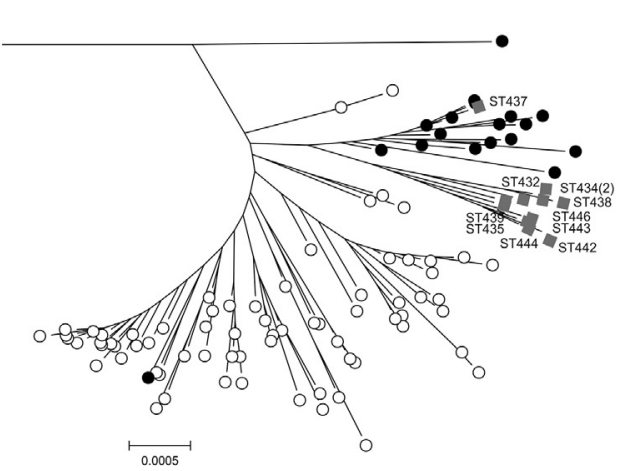

A. Borrelia afzelii

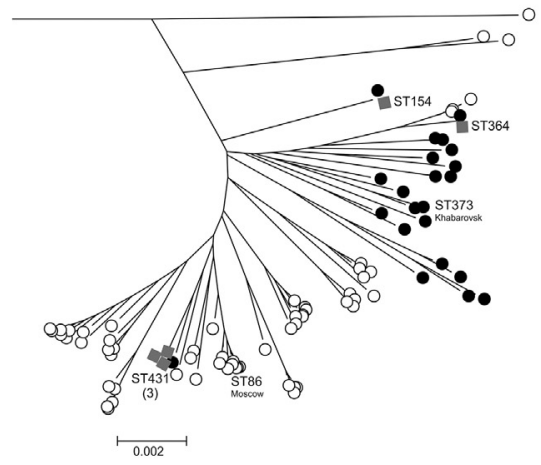

\section{Borrelia garinii}

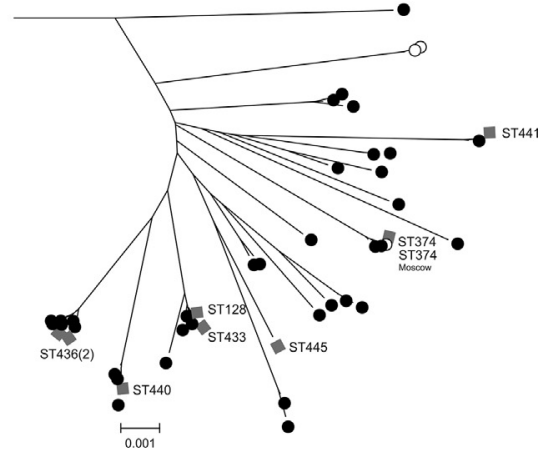

B. Borrelia bavariensis

Legend

Asian strains

European strains

(including European part of Russia)

Strains isolated

in the present study

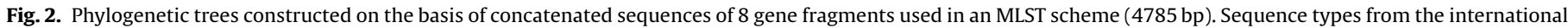

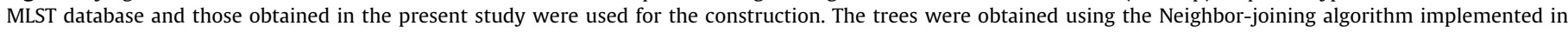
MEGA5. STs, isolated in Asia, Europe, and Russia (mostly in the present study) are shown in different colors.

it to cluster with B. garinii 20047 (Fig. 3), but not to form a separate clade.

B. afzelii isolates belonged mainly to the group VS461, homogeneity within the group reaching $99.5 \%$ and $94.1 \%$ based on MLST and IGS data, respectively. One isolate (Prm7050-12) belonged to the NT28 group, which was also originally discriminated on the basis of PCR-RFLP of rrf-rrl intergenic spacer (Masuzawa et al., 1996). As no strain of B. afzelii NT28 group has been typed using MLST scheme so far, performing a comparative analysis was not feasible. Despite the specific sequence of rrf-rrl IGS of B. afzelii NT28 group, it can be concluded that the distance between VS461 and NT28 groups is minimal based on the analysis of conserved housekeeping genes.

All MLST profiles of $B$. afzelii from Russia were obtained for the first time and do not refer to a previously known ST. B. afzelii from all regions were most closely related to strains from China and Japan. B. afzelii from the European part of Russia and Siberia formed a separate branch in the phylogenetic tree, while the Far Eastern isolate joined the clade formed by Chinese and Japanese strains (Fig. 2A). Strains that are close to the European B. afzelii were not found.

B. bavariensis was isolated mainly in Asia, with the exception of a few strains from Europe, including the European part of Russia (Fig. 2B). In our study, B. bavariensis strains were isolated in all studied regions of the country without forming a separate group of strains within the genospecies. Fig. 2B shows that they joined different clades within $B$. bavariensis regardless of geographic origin. The situation is similar for the $B$. garinii strains. Despite the fact that the strains of this species were found only in the city limits of Yekaterinburg and isolated from ticks collected in the same area, some of them are phylogenetically very distinct from each other (Fig. 2C).

\section{Discussion}

In Russia, Lyme disease was serologically confirmed for the first time in 1985, and in 1991, it was included in the official list of infectious diseases. Since that time, many studies have aimed to elucidate the clinical variants of the disease, and the features of the pathogen. By serological and molecular genetic methods, it was shown that 2 Borrelia genospecies are clinically significant in Russia: B. afzelii and B. garinii, with the latter being the most heterogeneous group. Firstly, B. garinii was divided into 5 serotypes (3-7) (Wilske et al., 1993), and later into 3 genomic groups (20047, NT29, and ChY13p) based on the PCR-RFLP (Postic et al., 1994; Li et al., 1998). In 2009, after the development of an MLST scheme, Borrelia serotype 4 was proposed to be a separate genospecies, $B$. bavariensis (Margos et al., 2009), which is also pathogenic to humans and associated with neurological manifestations of borreliosis. Doubtless, due to the introduction of a new classification, the question about the correlation between genomic groups and Borrelia genospecies was raised. Thus, in Russian scientific literature concerning the subject, $B$. bavariensis does not appear, although the name of this species is widely used in foreign publications, and it is recorded in the NCBI Taxonomy database as a separate species. However, the question is still under discussion, and B. bavariensis is absent in the conventional nomenclature of microorganisms as being an accepted species (Nolte, 2012). The analysis of Borrelia STs from Asia allows us to conclude that the majority of them are phylogenetically closer to B. bavariensis than to B. garinii 20047. For example, Takano et al. (2011) clearly distinguished 2 types within $B$. garinii, which clustered phylogenetically as clade A and clade B, the latter group including the prototype strain B. bavariensis PBi. In 2012, Margos et al. (2012) finally suggested that the genospecies B. bavariensis not only included PBi-like strains, but also strains of the NT29 genomic 


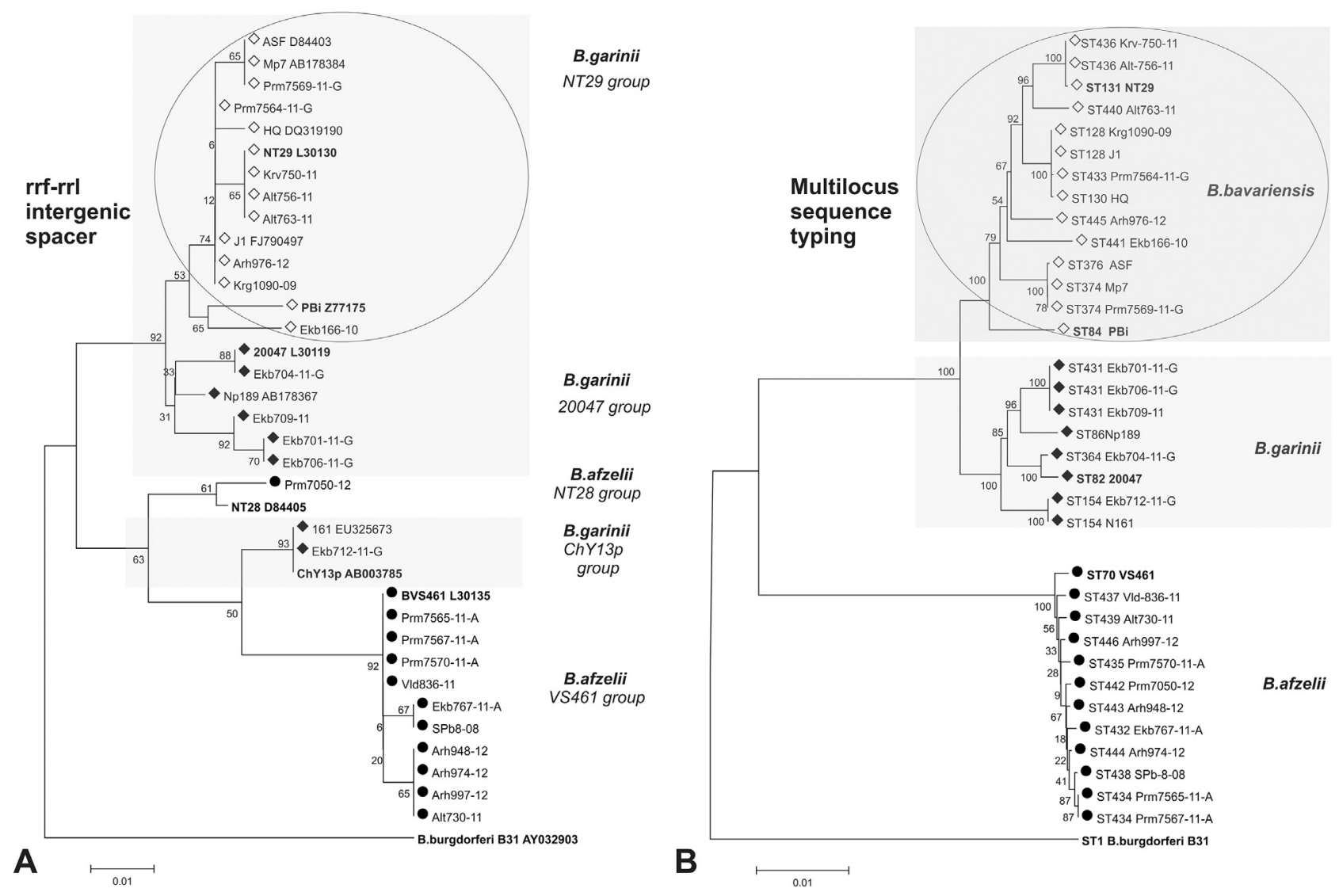

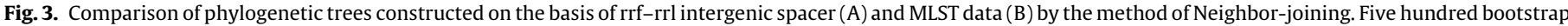

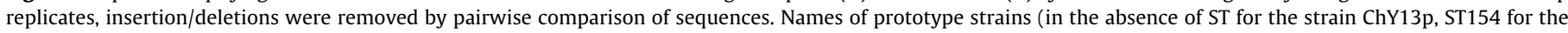
strain N161, belonging to the same group, was taken in the analysis) are given in bold. B. burgdorferi s.s. B31 was used as an outgroup.

group. Moreover, the formation of 2 subgroups within B. bavariensis appears to be a result of the adaptation to different vectors; i.e. PBilike strains, which are found only in Europe, seem to be transmitted by Ixodes ricinus (L., 1758) while Borrelia group NT29 is common in Asia and is transmitted mainly by I. persulcatus (Postic et al., 1997). The fact of a greater genetic diversity of Asian B. bavariensis, probably indicates an Asian origin of this species as recently proposed (Scholz et al., 2012). It was hypothesized that B. bavariensis was separated from a common ancestor with $B$. garinii by adaptation to a new host (rodents) and then adapted to I. ricinus in Europe (Margos et al., 2012).

The results obtained in the present study lend support to this hypothesis. Thus, the comparison of phylogenetic trees (Fig. 3) revealed that the genomic group NT29, formally differentiated by the characteristic restriction profile, corresponds to $B$. bavariensis. In this case, using PCR-RFLP (newly obtained and unregistered data), we can estimate the spatial distribution and prevalence of the 'new' genospecies, which is needed for phylogenetic and epidemiological studies. For example, the study of 227 B. garinii, isolated from 16 regions of Russia (Kaliningrad to Sakhalin) and analyzed by PCR-RFLP showed that 117 (51.6\%) belonged to the group 20047 (B. garinii); 108 (47.5\%) to a subgroup of NT29 (B. bavariensis); and $2(0.9 \%)$ to a subgroup of ChY13p (Nefedova et al., 2010). So, $B$. garinii and $B$. bavariensis in Russia are found in almost equal proportions. The latter genospecies was found in Russia almost from the beginning of LB epidemiology research (Postic et al., 1997).

Whilst B. garinii and B. bavariensis are found in Russia in almost equal proportions, the genomic group ChY13p, by contrast, is characterized by a very low prevalence. Despite the fact that the sequences of these strains form a distinct characteristic IGS
PCR-RFLP profile, MLST data show that they obviously belong to B. garinii 20047 (Fig. 3). There is a similar situation in the group $B$. afzelii NT28, whose IGS sequences have features in common with both $B$. afzelii and B. garinii (Li et al., 1998). Despite the fact that the analysis of IGS sequences allows us to consider the groups $B$. afzelii NT28 and B. garinii ChY13p as intermediate forms between 2 genospecies (Fig. 3), the MLST data do not support this hypothesis. Thus, we can assume that the sequence variations in the intergenic spacer do not reflect, objectively, evolutionary processes in Borrelia genospecies and cannot be the only criterion for dividing them into genomic groups. The causes of the emergence and fixation of specific mutations in the IGS and the possibility of recombination require further research.

Interesting questions about the ecology and geographic distribution of Borrelia genospecies were raised. Among the $6 \mathrm{~B}$. garinii serotypes only one (serotype 4 or B. bavariensis) is ecologically associated with rodents, while the others prefer birds as their main host. Thus, the preferential association with small mammals was shown for $B$. afzelii and $B$. bavariensis while birds seem to be the main reservoir host for $B$. garinii (Kurtenbach et al., 2002). Taking into account the much lower mobility of rodents, we can expect $B$. afzelii and $B$. bavariensis to have more distinct phylogeographic structures than $B$. garinii due to less active spatial mixing of strains. Our data generally support this hypothesis. In particular, it was shown that the $B$. afzelii populations showed pronounced structuring on the European scale and an almost complete absence of mixing of sequence types between different territories (Vollmer et al., 2011). The same was also observed for the Russian strains of B. afzelii, the Far Eastern strain Vld836-11 clustering with the Chinese and Japanese strains, and all other strains forming a separate 
clade (as previously no sequence type of B. afzelii from Russia was obtained), though related to the Asian strain. Thus, we suggest the Asian rather than European origin of $B$. afzelii isolated in Russia.

The 2 other species studied, B. bavariensis and B. garinii, showed evidence of spatial mixing of STs between geographic regions, which is most characteristic for B. garinii (Fig. 2B and C). This fact was revealed earlier (Margos et al., 2011; Vollmer et al., 2011).

Despite the relatively small sampling of strains used in this study, we were able to reveal some phylogeographic features of Borrelia in Russia, which was previously terra incognita. In order to better understand the phylogeography, evolution, and distribution of Borrelia, more isolates, including those from Russia, have to be obtained. The use of a reliable method to study the phylogeny and evolution based on MLST, clarifies and complements the existing classification as well as the standardized study protocol, and web resources created could became a basis for a global collaboration of scientists in studying the epidemiology of borreliosis.

\section{Acknowledgements}

We are indebted to V.L. Umpelev and B.A. Galishev for the material collection and to Dr Keith Chamberlain (Rothamsted Research) for his help in preparing the manuscript. The Russian Foundation of Basic Research (No. 12-04-31263) supported this project.

\section{References}

Enright, M.C., Spratt, B.G., 1999. Multilocus sequence typing. Trends Microbiol. 7, 482-487.

Kurtenbach, K., De Michelis, S., Etti, S., Schäfer, S.M., Sewell, H.S., Brade, V., Kraiczy, P., 2002. Host association of Borrelia burgdorferi sensu lato - the key role of host complement. Trends Microbiol. 10, 74-79.

Li, M., Masuzawa, T., Takada, N., Ishiguro, F., Fujita, H., Iwaki, A., Wang, H., Wang, J., Kawabata, M., Yanagihara, Y., 1998. Lyme disease Borrelia species in northeastern China resemble those isolated from far eastern Russia and Japan. Appl. Environ. Microbiol. 64, 2705-2709.

Livanova, N.N., Morozova, O.V., Morozov, I.V., Beklemishev, A.B., Cabello, F.C., Dobrotvorsky, A.K., 2003. Characterization of Borrelia burgdorferi sensu lato from Novosibirsk region (West Siberia, Russia) based on direct PCR. Eur. J. Epidemiol. $18,1155-1158$.

Margos, G., Castillo-Ramires, S., Hoen, A.G., 2012. Phylogeography of Lyme borreliosis-group spirochetes and methicillin-resistant Staphylococcus aureus. Parasitology 139, 1952-1965.

Margos, G., Gatewood, A.G., Aanensen, D.M., Hanincova, K., Terekhova, D., Vollmer, S.A., Cornet, M., Piesman, J., Donaghy, M., Bormane, A., Hurn, M.A., Feil, E.J., Fish, D., Casjens, S., Wormser, G.P., Schwartz, I., Kurtenbach, K., 2008. MLST of housekeeping genes captures geographic population structure and suggests a European origin of Borrelia burgdorferi. Proc. Natl. Acad. Sci. U. S. A. 105, 8730-8735.

Margos, G., Hojgaard, A., Lane, R.S., Cornet, M., Fingerle, V., Rudenko, N., Ogden, N., Aanensen, D.M., Fish, D., Piesman, J., 2010. Multilocus sequence analysis of Borrelia bissettii strains from North America reveals a new Borrelia species, Borrelia kurtenbachii. Ticks Tick Borne Dis. 1, 151-158.

Margos, G., Vollmer, S.A., Cornet, M., Garnier, M., Fingerle, V., Wilske, B., Bormane, A., Vitorino, L., Collares-Pereira, M., Drancourt, M., Kurtenbach, K., 2009. A new Borrelia species defined by multilocus sequence analysis of housekeeping genes. Appl. Environ. Microbiol. 75, 5410-5416.

Margos, G., Vollmer, S.A., Ogden, N.H., Fish, D., 2011. Population genetics, taxonomy, phylogeny and evolution of Borrelia burgdorferi sensu lato. Infect. Genet. Evol. $11,1545-1563$.

Masuzawa, T., Komikado, T., Iwaki, A., Suzuki, H., Kaneda, K., Yanagihara, Y., 1996. Characterization of Borrelia sp. isolated from Ixodes tanuki, I. turdus, and I. columnae in Japan by restriction fragment length polymorphism of $\operatorname{rrf}(5 \mathrm{~S})-\operatorname{rrl}(23 \mathrm{~S})$ intergenic spacer amplicons. FEMS Microbiol. Lett. 142, 77-83.

Nefedova, V.V., Korenberg, E.I., Gorelova, N.B., 2010. Genetic variants of Borrelia garinii, a widely spread Eurasian pathogen of ixodic tick borreliosis. Mol. Gen. Mikrobiol. Virusol., 7-12 (in Russian).

Nolte, O., 2012. Nucleic acid amplification-based diagnostic of Lyme (neuro-) borreliosis - lost in the jungle of methods, targets, and assays? Open Neurol. J. 6, 129-139.

Ornstein, K., Barbour, A.G., 2006. A reverse transcriptase-polymerase chain reaction assay of Borrelia burgdorferi 16S rRNA for highly sensitive quantification of pathogen load in a vector. Vector Borne Zoonotic Dis. 6, 103-112.

Postic, D., Assous, M.V., Grimont, P.A., Baranton, G., 1994. Diversity of Borrelia burgdorferi sensu lato evidenced by restriction fragment length polymorphism of $\operatorname{rrf}(5 \mathrm{~S})-\mathrm{rrl}$ (23S) intergenic spacer amplicons. Int. J. Syst. Bacteriol. 44, $743-752$.

Postic, D., Korenberg, E., Gorelova, N., Kovalevski, Y.V., Bellenger, E., Baranton, G., 1997. Borrelia burgdorferi sensu lato in Russia and neighbouring countries: high incidence of mixed isolates. Res. Microbiol. 148, 691-702.

Scholz, H.C., Margos, G., Derschum, H., Speck, S., Tserennorov, D., Erdenebat, N., Undraa, B., Enkhtuja, M., Battsetseg, J., Otgonchimeg, C., Otgonsuren, G., Nymadulam, B., Römer, A., Thomas, A., Essbauer, S., Wölfel, R., Kiefer, D., Zöller, L., Otgonbaatar, D., Fingerle, V., 2012. High prevalence of genetically diverse Borrelia bavariensis-like strains in Ixodes persulcatus from Selenge Aimag, Mongolia. Ticks Tick Borne Dis. 4, 89-92.

Sullivan, C.B., Diggle, M.A., Clarke, S.C., 2005. Multilocus sequence typing: data analysis in clinical microbiology and public health. Mol. Biotechnol. 29, 245-254.

Takano, A., Nakao, M., Masuzawa, T., Takada, N., Yano, Y., Ishiguro, F., Fujita, H., Ito, T., Ma, X., Oikawa, Y., Kawamori, F., Kumagai, K., Mikami, T., Hanaoka, N., Ando, S., Honda, N., Taylor, K., Tsubota, T., Konnai, S., Watanabe, H., Ohnishi, M., Kawabata, H., 2011. Multilocus sequence typing implicates rodents as the main reservoir host of human-pathogenic Borrelia garinii in Japan. J. Clin. Microbiol. 49, 2035-2039.

Tamura, K., Peterson, D., Peterson, N., Stecher, G., Nei, M., Kumar, S., 2011. MEGA5: molecular evolutionary genetics analysis using maximum likelihood, evolutionary distance, and maximum parsimony methods. Mol. Biol. Evol. 28, 2731-2739.

Vollmer, S.A., Bormane, A., Dinnis, R.E., Seelig, F., Dobson, A.D., Aanensen, D.M., James, M.C., Donaghy, M., Randolph, S.E., Feil, E.J., Kurtenbach, K., Margos, G., 2011. Host migration impacts on the phylogeography of Lyme Borreliosis spirochaete species in Europe. Environ. Microbiol. 13, 184-192.

Wilske, B., Preac-Mursic, V., Göbel, U.B., Graf, B., Jauris, S., Soutschek, E., Schwab, E. Zumstein, G., 1993. An OspA serotyping system for Borrelia burgdorferi based on reactivity with monoclonal antibodies and OspA sequence analysis. J. Clin. Microbiol. 31, 340-350.

Yastrebov, V.K., Rudakov, N.V., Shpynov, S.N., 2012. Transmissive tick-borne natural focal infections in the Russian Federation: trends of the epidemiological process, actual prophylaxis problems. Siberian Med. J. 4, 91-93. 